\title{
Transbronchial and transesophageal fine- needle aspiration using a single ultrasound bronchoscope in the diagnosis of locoregional recurrence of surgically-treated lung cancer
}

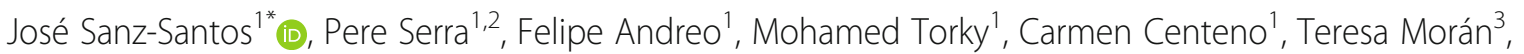
Enric Carcereny ${ }^{4}$, Esther Fernández ${ }^{4}$, Samuel García-Reina ${ }^{4}$ and Juan Ruiz-Manzano ${ }^{1}$

\begin{abstract}
Background: The present study sought to evaluate the usefulness of EBUS-TBNA in the diagnosis of locoregional recurrence of lung cancer in a cohort of lung cancer patients who were previously treated surgically, and describe our initial experience of EUS-B-FNA in this clinical scenario.

Methods: We retrospectively studied the clinical records of all patients with a previous surgically-treated lung cancer who were referred to our bronchoscopy unit after suspicion of locoregional recurrence. The diagnostic sensitivity, specificity, positive predictive value (PPV), negative predictive value (NPV) and overall accuracy of EBUS-TBNA for the diagnosis of locoregional recurrence were evaluated.

Results: Seventy-three patients were included. EBUS-TBNA confirmed malignancy in 40 patients: 34 confirmed to have locoregional recurrence, six had metachronous tumours. Of the 33 patients with non-malignant EBUS-TBNA; 2 had specific non-malignant diseases, 26 underwent radiological follow up and 5 patients underwent surgery. Of the 26 patients who had radiological follow up; 18 remained stable, three presented thoracic radiological progression and 5 presented extrathoracic progression. Of the 5 patients who underwent surgery; 3 had metachronous tumours, one confirmed to be a true negative and one presented nodal invasion. Seven patients underwent EUS-B-FNA, four of them confirmed to have recurrence. The sensitivity, specificity, NPV, PPV and overall accuracy of EBUS-TBNA for the diagnosis of locoregional recurrence were 80.9, 100, 69.2, 100 and 86.6\% respectively.
\end{abstract}

Conclusions: EBUS-TBNA is an accurate procedure for the diagnosis of locoregional recurrence of surgically-treated lung cancer. EUS-B-FNA combined with EBUS-TBNA broads the diagnostic yield of EBUS-TBNA alone.

Keywords: Lung cancer, Recurrence, Surgically-treated, Endobronchial Ultrasound, Transbronchial needle aspiration, Endoscopic ultrasound, Fine needle aspiration

\footnotetext{
* Correspondence: jsanzsantos@gmail.com

${ }^{1}$ Pulmonology Department, Hospital Germans Trias i Pujol, Carretera de

Canyet S/N. 08916, Badalona, Barcelona, Spain

Full list of author information is available at the end of the article
} 


\section{Background}

Lung cancer is the leading cause of cancer-related mortality worldwide [1]. Surgery resection with a curative intent is the most effective treatment for early stage non-small- cell lung cancer (NSCLC). However, even after complete surgical resection, the rate of recurrence for stages I to III of NSCLC range from 30 to 70\% [2] with a high incidence of recurrence during the first 2 years. Based on this concern, published practice guidelines recommend multidisciplinary clinical and radiographic follow up of patients with resected lung cancer [3]. More specifically, the American College of Chest Physicians recommends that in patients who underwent a curative-intent surgical resection of a NSCLC, a chest computer tomography (CT) should be performed every 6 months for the first 2 years after resection and every year thereafter. In many cases, surgical treatment must be combined with adjuvant chemotherapy and/or radiotherapy which can cause inflammation and fibrosis of the mediastinum. Furthermore, most of lung cancer patients are smokers who may have Chronic Obstructive Pulmonary Disease (COPD) or other inflammatory lung disorders which may result in infectious complications. These circumstances such as inflammation, fibrosis and infectious complications could be presented as both lung parenchymal abnormalities and mediastinal nodal enlargement in thoracic CT that can be misdiagnosed as regional recurrence [4]. Thereby, mediastinal and/or hilar nodal enlargement in the thoracic CT during follow up are common features that usually represent a challenge for the clinician. Positron emission tomography with computer tomography (PET/CT) has been used as a diagnostic tool for recurrence, with a high sensitivity value $[5,6]$. However, both CT and PET/CT have shown high false-positive rates and therefore histological confirmation is mandatory to rule out regional recurrence.

Mediastinoscopy has been demonstrated to be useful in the assessment of recurrence in patients with previous surgically-treated lung cancer [7]. However, mediastinoscopy becomes more difficult, unsafe and useless after previous thoracic surgery, especially in patients who have been treated with induction radiotherapy. Furthermore, recurrence can lay on hilar nodes that are not amenable for biopsy by means of mediastinoscopy.

Endobronchial ultrasound-guided transbronchial needle aspiration (EBUS-TBNA) is a minimum invasive procedure currently proposed as the first choice in the mediastinal nodal staging of lung cancer [8]. Adding endoscopic ultrasound fine needle aspiration (EUS-FNA) to EBUS-TBNA has revealed higher diagnostic yield than using EBUS-TBNA alone, due to the complementary access for different nodal stations by each technique [9]. The evaluation of mediastinal nodes from the esophagus using a convex probe EBUS (EUS-B-FNA) associated to EBUS-TBNA has proven to be useful, with figures similar to those using combination of EUS and EBUS-TBNA [10]. The applications of EUS-B-FNA further than lung cancer staging have been barely investigated [11-13].

Some previous studies have demonstrated the usefulness of EBUS-TBNA in the diagnosis of recurrence in patients with previously treated lung cancer [14-18]. However, some of these studies included short series of subjects or included patients not surgically treated. The aim of our study was to evaluate the usefulness of EBUS-TBNA in the diagnosis of locoregional lung cancer recurrence in a larger cohort of subjects entirely composed of surgically-treated patients and describe our initial experience of EUS-B-FNA in this clinical scenario.

\section{Methods \\ Patients}

We conducted a single-center, retrospective study that included all patients with a previous surgically-treated lung cancer who were referred to our bronchoscopy unit after suspicion of locoregional recurrence from January 2006 to October 2014. Recurrence suspicion was based on hilar or mediastinal lymph node enlargement on CT scan ( $>10 \mathrm{~mm}$ in the short axis on CT) and/or abnormal nodal fludeoxyglucose (FDG) avidity on PET-CT during follow up with or without pulmonary node/s or mass/es. The medical records of all patients were reviewed and clinical characteristics were introduced in a database.

\section{EBUS-TBNA}

EBUS was performed at an out-patient setting using a flexible bronchoscope [BFUC180F, Olympus Optical Co Ltd., Tokyo, Japan] with a distal probe capable of producing linear parallel scans of both mediastinal and peribronchial tissues also a working channel suited for the performance of TBNA under direct ultrasound guidance. Local anesthesia and sedation were achieved using topical lidocaine spray and intravenous midazolam, propofol and/or fentanyl in accordance with the standard recommendations [19]. Identified mediastinal and lobar nodes with short-axis diameter of $5 \mathrm{~mm}$ or more were targeted under direct ultrasound visualization with a 22-gauge cytology needle specially designed for EBUS-TBNA [NA-201SX-4022, Olympus Optical Co Ltd.]. After passing through the bronchoscope channel, the needle was pushed out of the sheath and inserted into the tracheal or bronchial wall under ultrasound guidance. At the target tissue, the needle tip was located, and then it was pushed forth and back with application of negative pressure using a syringe (with $10-\mathrm{mL}$ suction) connected to the proximal end of the catheter. Finally, the suction was ceased while withdrawing the needle out of the target structure. Samples were labeled according to their 
origin, whether it was a normal node showing lymphocytic cells and no neoplastic cells, or a metastatic node showing neoplastic cells. Aspirates containing only isolated dysplastic, bronchial, esophageal or blood cells or necrotic tissue were considered inadequate. Nodal sampling was targeted and nodes with high suspicion of malignancy (enlarged on CT scan and/or abnormal FDG avidity) were firstly sampled. If rapid on site examination confirmed malignancy the procedure was then finished. In case of a non malignant result, suspicious nodes were sampled three times before ruling out malignancy and a complete systematic sampling (including both lower paratracheal, and subcarinal stations) was performed.

\section{EUS-B-FNA}

In cases with lesions that were inaccessible through EBUS-TBNA (nodal stations 5, 8 or 9) the patient underwent directly EUS-B-FNA. In cases with lesions accessible through EBUS-TBNA the patient firstly underwent EBUS-TBNA. If the lesion was partially visible through EBUS-TBNA and located in a station accessible through EUS-B-FNA then the patient underwent EUS-BFNA in a single-session procedure. EUS-B-FNA was performed guiding the bronchoscope through the pharynx and advanced into the esophagus under gentle pressure. The sampling method did not differ to that previously described for EBUS-TBNA.

\section{Pathology}

The aspirated material in the needle was recovered and the specimens were placed on slides and fixed with $95 \%$ ethanol. The slides were stained for one minute with haematoxylin for rapid on-site evaluation. Papanicolau staining with orange A and eosin was done later in the pathology laboratory. The cytologist classified satisfactory nodal samples as "normal tissue negative for malignancy" when the sample contained 40 lymphocytes per high-power field in cellular areas of the smear and/or clusters of pigmented macrophages and no neoplastic cells, or as "metastatic" when recognizable groups of malignant cells were present [20]. Nodes containing only isolated dysplastic, bronchial, esophageal or blood cells or necrotic tissue were considered as non-representative of the targeted structure, and were classified as inadequate. Cell blocks were obtained and processed from the specimens recovered whenever extra material was available after the preparation of a minimum of four slides.

\section{Definitions}

Cases in which EBUS-TBNA demonstrated malignant nodes with the same histology as the previous treated lung cancer were considered as recurrence (true positive) and no confirmatory tests were required. Cases in which EBUS-TBNA demonstrated specific benign diseases were considered as true negative and no confirmatory tests were carried out. Cases where EBUS-TBNA demonstrated lymphocytes without malignant cells (negative EBUSTBNA) underwent confirmatory surgery as a "gold standard" or radiological follow up. Negative EBUSTBNA was considered true negative if the surgical procedures did not demonstrate nodal malignancy or if remained stable during radiological follow up for 12 months. Negative EBUS-TBNA were considered false negative if the surgical procedures demonstrated nodal malignancy or if radiological progression was proved by CT. Patients that presented with extrathoracic progression that required chemotherapy and/or could not complete 12 months of radiological follow up were also considered false negative. Although diagnostic yield of EBUS-TBNA for global malignancy included patients with metachronous tumours, outcomes of these patients were not used in evaluating diagnostic yield of EBUS-TBNA for locoregional recurrence. Metachronous tumours were defined by Martini et al. [21] as follows: 1) Different histological type from the primary tumour or 2) Same histological type if: a) Free interval between tumours is at least 2 years or b) Origin from carcinoma in situ or c) Second cancer in different lobe or lung, but: i) No carcinoma in lymphatics common to both, ii) No extrapulmonary metastases at time of diagnosis.

\section{Statistical analysis}

Data was entered into a database and analyzed using SPSS software, version 18.0 [Chicago, IL, USA]. Categorical variables were expressed as absolute and relative frequencies, continuous variables as means and standard deviations (SD) and non-normally distributed data as medians and interquartile ranges (IQR). The diagnostic sensitivity, specificity, positive predictive value (PPV), negative predictive value (NPV) and overall accuracy of EBUS-TBNA were calculated according to standard definitions for diagnosis of both locoregional recurrence and global malignancies, including metachronous tumors, in patients with previous surgically-treated lung cancer.

\section{Results}

Seventy-three patients were included. Patient characteristics are shown at Table 1. Previous histological subtypes of lung cancer were predominantly squamous cell-carcinoma and adenocarcinoma and the majority of patients had undergone previous lobectomy. Most of the patients had a previous systematic mediastinal nodal dissection; the median number of dissected nodes was 14.5 per patient. Six patients had a previous staging mediastinoscopy and 20 patients had undergone EBUS-TBNA before surgery. Primary therapy for lung cancer included surgical resection alone in 47 patients; surgical resection followed by chemotherapy in 16; surgical resection followed by 
Table 1 Patients' characteristics

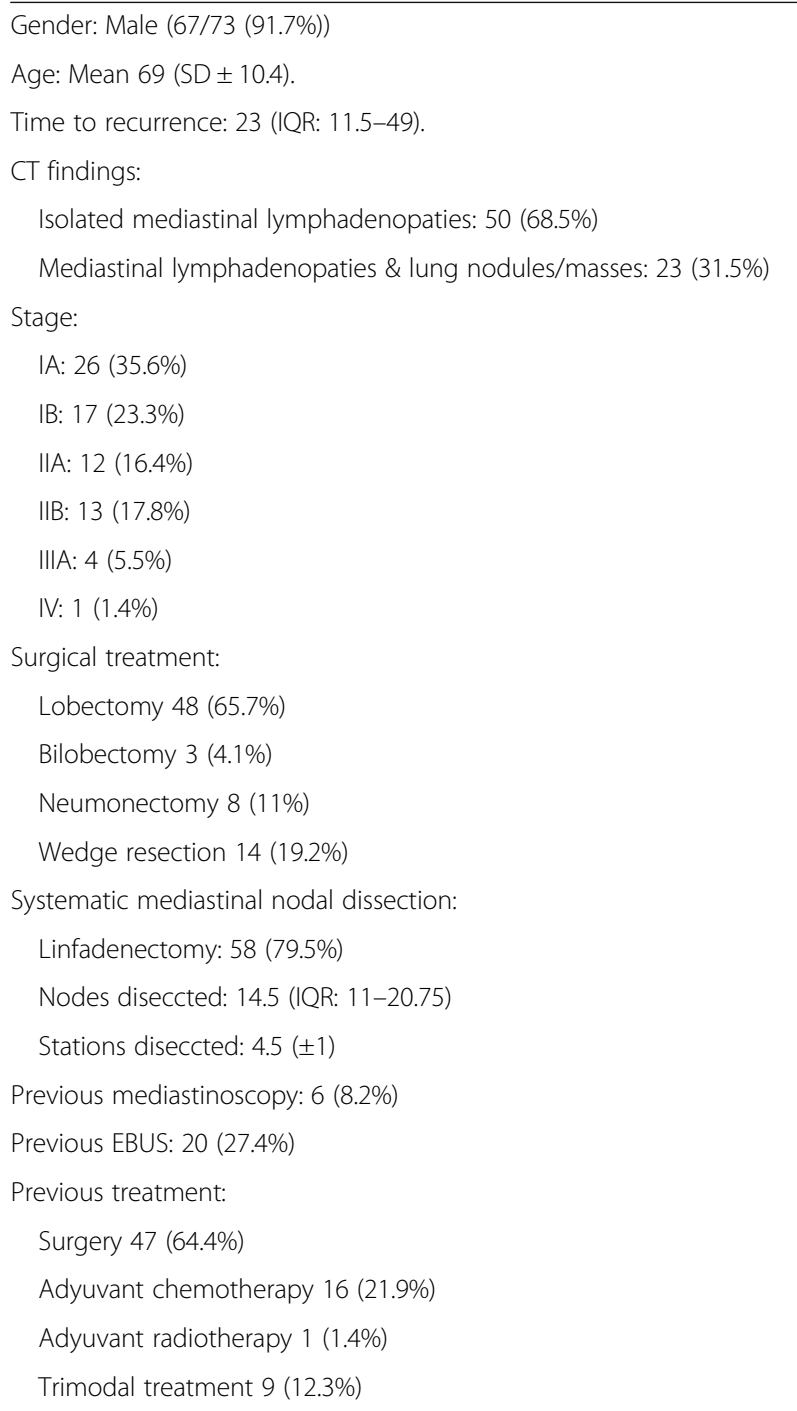

radiotherapy in one patient and induction chemo radiotherapy followed by surgical resection in 9 patients. One patient was diagnosed as stage IV but could benefit for surgical treatment after resection of the single extrathoracic metastasis. The median time between primary surgical treatment and recurrence suspicion was 23 months. CT findings were mediastinal nodal enlargement alone in 50 patients and mediastinal nodal enlargement with lung nodes/masses in 23 patients.

EBUS-TBNA confirmed malignancy in 40 patients while in the remaining 33 did not show malignancy (Fig. 1). The entire 40 patient with malignant nodes were presented with the same histological type as the previous treated lung cancer. However, six patients were considered as metachronous disease instead of recurrence. Of the 34 patients confirmed to have recurrence by EBUS-TBNA, 15 patients underwent chemotherapy, 3 radiotherapy, 14 concurrent chemoradiotherapy and 2 patients received best supportive care. In most of the cases, nodal recurrence affected ipsilateral hilar or mediastinal nodes and, in $20 \%$ recurrence affected contralateral mediastinum (N3). Eleven malignant lymph nodes (8 hilars, 3 paraesophageal stations), collected from 10 patients who resembled $29.4 \%$ of all recurrent patients diagnosed by EBUS-TBNA, were out of the reach of mediastinoscopy.

Of the 33 patients with non-malignant EBUS-TBNA, two had a specific non-malignant disease diagnosed by EBUS-TBNA (1 nodal tuberculosis, 1 foreign body reaction). Of the other 31 patients, 26 patients underwent radiological follow up and 5 patients underwent surgery. Of the 5 patients that underwent surgery one patient with isolated lymphadenopathies underwent mediastinoscopy which showed normal lymph tissue and thereby was considered as true negative; the other 4 patients that presented node or mass associated to the nodal enlargement underwent lobectomy: 3 patients had a final diagnosis of metachronous lung cancer without nodal involvement while one other patient presented a $\mathrm{N} 1$ positive interlobar node in the lobectomy resection sample and thus was considered as a false negative of the EBUS-TBNA.

Of the 26 patients who had radiological follow up 18 patients remained stable after 12 months, three presented with thoracic radiological progression and 5 patients presented with extrathoracic progression and died before completing 12 months of follow-up and thereby were considered false negative.

Seven patients underwent EUS-B-FNA because the lesion was difficult to be accessed (one 2R station node, one $4 \mathrm{~L}$ station node) or inaccessible for EBUS (five paraesophageal (8) stations) (Table 2, Fig. 2). Of the 2 patients with a lesion accessible by means of EBUSTBNA both had an EBUS-TBNA before the EUS-B-FNA in a single-session procedure while the 5 patients with lesions not reachable by means of EBUS-TBNA directly underwent EUS-B-FNA. EUS-B-FNA confirmed the recurrence in four cases. There were no major complications related to EBUS-TBNA.

The sensitivity, specificity, negative predictive value, positive predictive value and overall accuracy of EBUSTBNA for the diagnosis of locoregional recurrence in patients with previous surgically-treated lung cancer were $80.9,100,69.2,100$ and $86.6 \%$ respectively. The sensitivity, specificity, negative predictive value, positive predictive value and overall accuracy of EBUS-TBNA for the diagnosis of global malignancy, including metachronous tumours, in patients with previous surgically-treated lung cancer were $81.6,100,72.7,100$ and $87.6 \%$ respectively.

\section{Discussion}

Locoregional recurrence represents a significant problem in lung cancer, which corresponds to a quarter of recurrences after surgery [22]. Reported rates of locoregional 


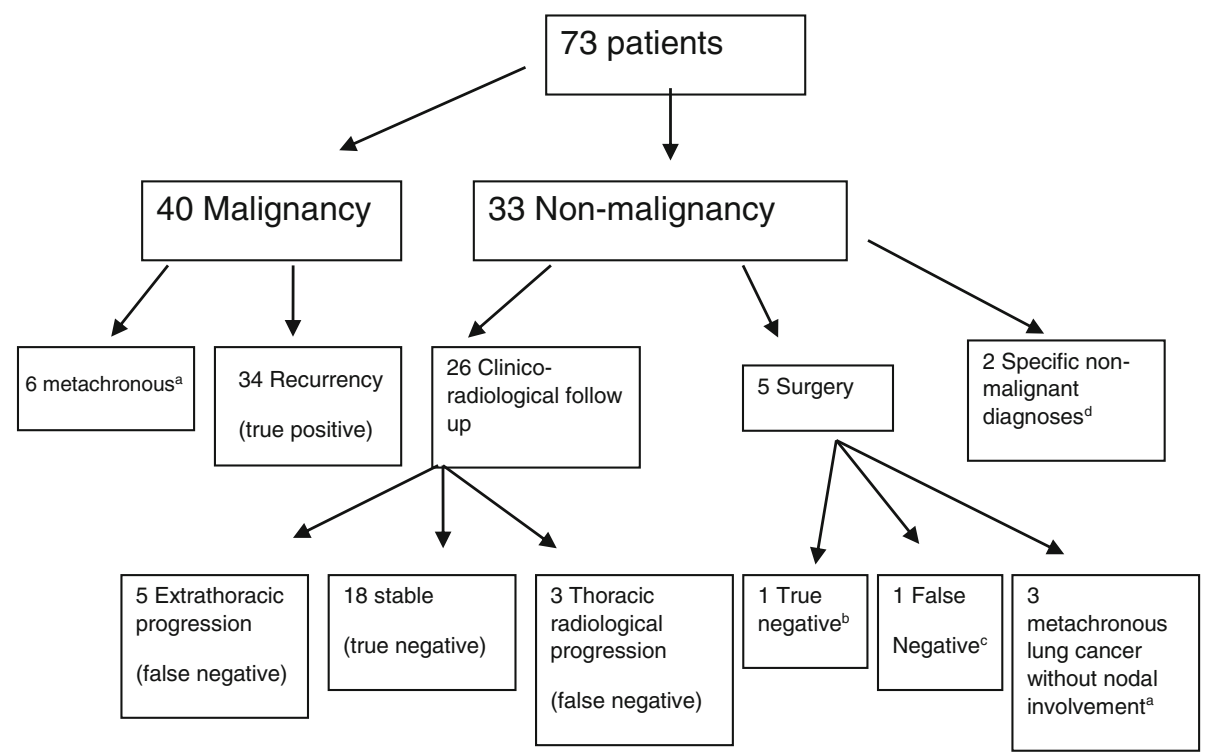

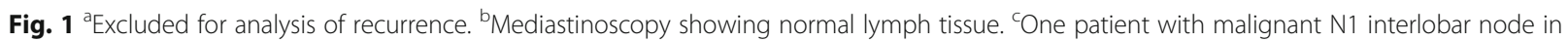
the resection specimen. ${ }^{\mathrm{d}}$ One tuberculosis, one foreign body reaction

recurrence of NSCLC after surgery vary widely in literature due to the heterogeneity of the studies, as some of them including small sample sizes, variable disease stages, differences in follow up time and unclear definitions of recurrence versus metachronous tumours. Locoregional recurrences typically occur rapidly, as nearly $90-95 \%$ of all local recurrences develop during

Table 2 EBUS procedure

\begin{tabular}{|c|c|}
\hline Total nodes samp & \\
\hline Nodes sampled (p & \\
\hline Stations sampled & \\
\hline Malignant nodes ${ }^{a}$ : & \\
\hline Mediastinal: 37 & \\
\hline $2 \mathrm{~L}: 1$ & 2R: 1 \\
\hline 4R: 7 & $4 \mathrm{L:} 11$ \\
\hline 7: 14 & \\
\hline $8 \mathrm{~L}: 2$ & 8 L: 1 \\
\hline Hilar: 8 & \\
\hline 10 L: 1 & 10R: 2 \\
\hline 11 L: 2 & 11R: 3 \\
\hline Mean size ${ }^{\text {b: }} 14.8(I$ & \\
\hline Esophagus (EUS-B & \\
\hline $\mathrm{N}$ in recurrence $(3$ & \\
\hline N1: 5 (14.7\%) & \\
\hline N2: 22 (64.7\%) & \\
\hline N3: 7 (20.6\%) & \\
\hline
\end{tabular}

In patients with recurrence diagnosed by EBUS-TBNA

b In mm (short-axis diameter) the first five years after the initial surgery [23]. Pathological stage, surgical technique (wedge resection or segmentectomy versus full lobar resection) and patients' characteristics have been demonstrated to be independent predictors of recurrence. Although postoperative surveillance programs have not established a survival benefit, most guidelines recommend follow up of patients after curative-intent surgery. In our series, half of the patients were presented with nodal local recurrence, $6.8 \%$ extrathoracic metastases and $12 \%$ metachronous lung cancer, confirming that the likelihood of malignancy in these patients is high and thus the diagnosis and clinical-decision making cannot rely only on image-based explorations.

Before EBUS-TBNA, mediastinoscopy was the most used diagnostic method as an invasive approach to explore the mediastinum. However, few studies have focused on the role of mediastinoscopy in the diagnosis of locoregional recurrence. The concept of "complex" mediastinum refers to an altered fibrotic mediastinum secondary to previous thoracic surgery (primary mediastinoscopy or nodal dissection) or induction therapy [24]. Although some studies performed by expert surgeons in referral hospitals have shown good accuracy of remediastinoscopy, with complication rates similar to primary mediastinoscopy [25], remediastinoscopy is not widely used due to concerns about safety and usefulness [26]. In our series, one third of the patients had concurrent radiotherapy and/or chemotherapy and 7 patients had a previous mediastinoscopy. Regarding the diagnosis of local lymph node recurrence, only one patient required mediastinoscopy after an EBUS. This particular patient 

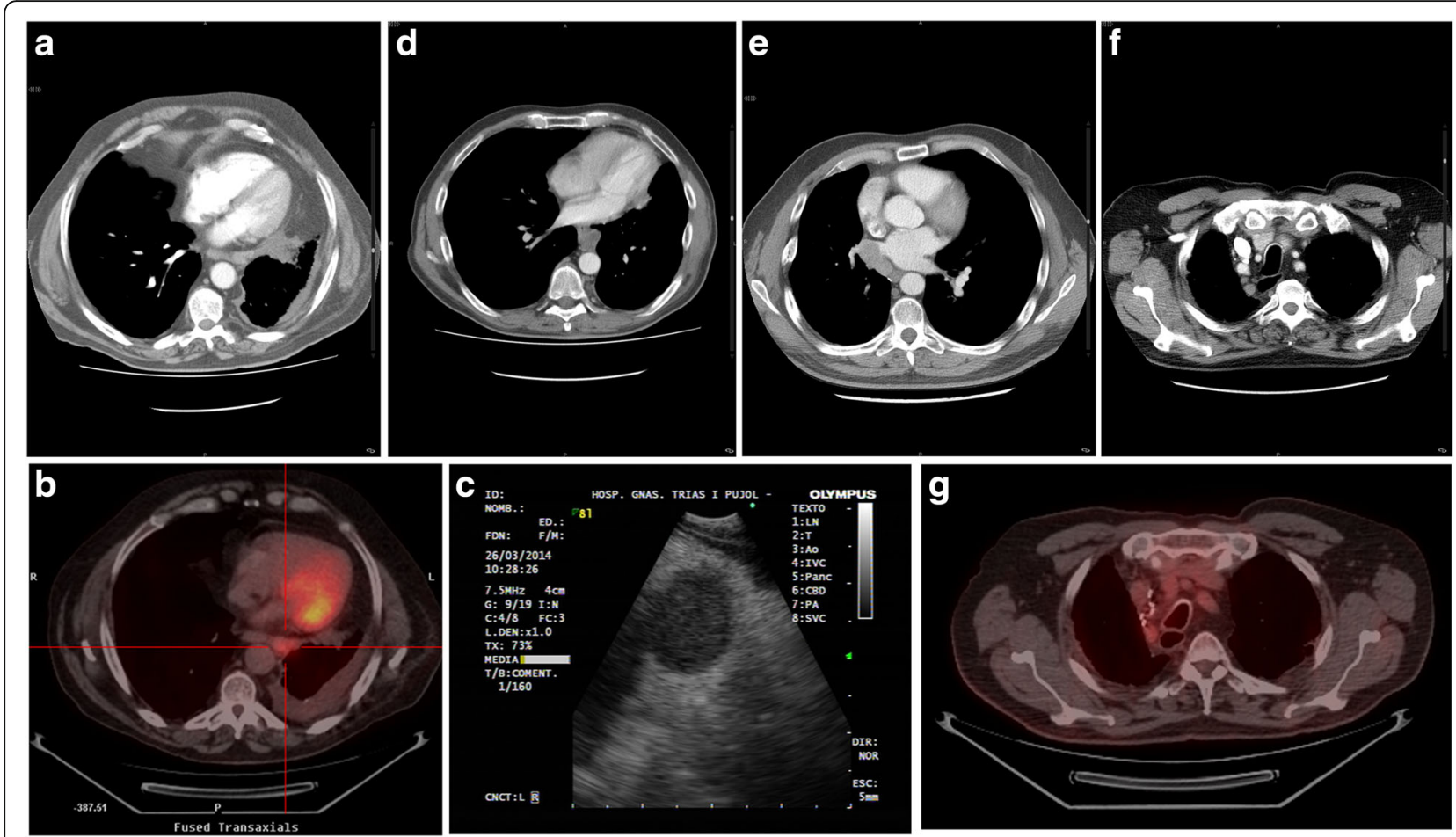

Fig. 2 Four cases of recurrence diagnosed by EUS-B-FNA: Case 1 (a, b, c): a 68-years old man with a stage IIA squamous-cell carcinoma in LLL treated by means of lobectomy presented a pleural effusion on the first CT control 6 months after the surgery, the PET/CT showed a high uptake on a 8 left station node, an EUS-B-FNA confirmed recurrence. Case 2 (d): a 55-years old man with a previous stage IA NSCLC-NOS in RUL treated by means of wedge resection presented an 8 left lymphadenopathy on the first CT control 6 months after the surgery. Case 3 (e): a 40 -years old man with a carcinoid on middle lobe treated by means of RLL and ML bilobectomy presented a mass/nodal enlargement on right 8 station that invaded the pulmonary veins, the diagnosis was consistent with atypical carcinoid. Case 4 (f, $\mathbf{g}$ ): a 71-year old man with a stage IIA adenocarcinoma in RUL treated by means of lobectomy presented a 2R adenopathy in a CT 21 months before the surgery. Although being a paratracheal node the approach was easier through the esophagus

neither underwent previous mediastinoscopy nor received neo/adjuvant therapy. The mediastinoscopy showed no malignancy and the patient remained stable in subsequent radiological follow up. In our series, almost one third of the patients with recurrence confirmed by EBUS-TBNA presented with nodes out of the reach of mediastinoscopy: 3 patients presented with paraesophageal lesions and 7 patients had hilar lesions.

One of the advantages of EBUS-TBNA is its ability to sample hilar nodes. Probably this advantage has not been properly emphasized. One possible explanation is that EBUS-TBNA has been mainly used for mediastinal staging of lung cancer where hilar involvement is not relevant enough since contralateral hilar N3 involvement without N3 mediastinal affection is not very frequent and the distinction between N0/N1, although has importance in the prognosis and the choice of treatment, rarely affects the surgical indication. Nevertheless, the evaluation of $\mathrm{N} 1$ nodes is crucial in patients undergoing sublobar resection or local tissue-sparing treatments such as brachytherapy, radiofrequency ablation and stereotactic body radiation. These are the only therapeutic options to many patients who cannot benefit from conventional surgical intervention, including those with previous surgical treatment like in our series. In our study the only surgical false negative case presented with $\mathrm{N} 1$ interlobar node. The reported sensibility for EBUS-TBNA in the diagnosis of N1 disease is lower than that described for N2 staging [27], mainly because the size of the convex probe EBUS in many cases does not permit to reach $\mathrm{N} 1$ nodes beyond station 11. However, this issue could be solved with the development of new thinner convex probe EBUS. Recently, Wada et al. [28] described their first experience with a new thin convex probe echobronchoscope [BFY0046 Olympus Medical Systems Corp] with a thinner tip $(5.9 \mathrm{~mm})$ and a larger bending angle than conventional convex probe ecobronchoscopes. These authors reported an improved accessibility to the distal airways in a porcine model, suggesting that thinner echobronchoscopes would increase the diagnostic yield of N1 nodes (especially those beyond the hilum) and peripheral nodes/masses.

Endoscopic ultrasound with a convex probe ultrasonic bronchoscope (EUS-B-FNA) was first described in 2009 [10]. EUS-B-FNA broads the diagnostic yield of EBUS- 
TBNA alone because it allows the approach of nodal stations that are not in contact with the tracheobronchial wall (stations 5 and lower mediastinal stations: 8, 9) and also increases the access to nodes that could be better achieved through the esophagus. Moreover EUS-B-FNA is also better tolerated and safer in patients with respiratory impairment. Although these advantages, EUS-BFNA requires a learning period before systematically employed, as the endobronchial references are lost on the white light image and the ultrasonographic anatomy boundaries vary from the endobronchial view. In our series, ten percent of the patients underwent EUS-BFNA, and 4 patients $(12.9 \%)$ with recurrence diagnosed by EBUS-TBNA were exclusively diagnosed by EUS-BFNA (3 in paraesophageal stations and one in $2 \mathrm{R}$ station). In our study, some nodes although located on subcarinal or paratracheal stations, were more easily reached by the esophagus (Fig. 2) due the post-surgical changes in the mediastinal architecture. Few studies have described the usefulness of EUS-B-FNA apart from lung cancer staging. Szlubowski et al. [11] published their experience with EUS-B-FNA combined with EBUS-TBNA in the lung cancer restaging after induction therapy and other two studies described the usefulness of EUS-B-FNA in the sampling of left adrenal gland in patients with lung cancer [12, 13]. To our knowledge this is the first study in which EUS-BFNA has been used in the diagnosis of locoregional lung cancer recurrence.

Although the interpretation of the visualized mediastinal structures after surgery is more difficult than in a naïve mediastinum, our study demonstrates excellent diagnostic performance of EBUS-TBNA in the diagnosis of locoregional recurrence of patients with surgically treated lung cancer. Our results are similar to those reported for new developed lung cancer staging and also comparable to those to previous studies that included shorter series of surgically-treated patients. Yamamoto et al. [17] in a series of 40 surgically-treated patients showed a sensitivity and NPV of $100 \%$, while Han et al. [16] in a series of 42 surgically-treated patients showed a sensitivity of $94.3 \%$ and a NPV of $77.8 \%$. Other studies that included non-surgically-treated patients $[14,15,18]$ also demonstrated a high diagnostic accuracy of EBUSTBNA in the diagnosis of lung cancer recurrence. Thus, changes in the mediastinal anatomy in a post-surgical mediastinum do not affect the diagnostic yield of EBUSTBNA procedure. Remarkably, in another situation of "complex" mediastinum, following induction therapy, EBUS-TBNA showed lower diagnostic value in restaging of lung cancer $[29,30]$. This is probably not due to the procedure itself, but because of complexity in the interpretation of the pathological samples. It has been previously reported that aspirates from malignant nodes treated with chemotherapy may content less cellular burden that could have necrotic tissue, making the pathologic interpretation more difficult.

\section{Conclusions}

EBUS-TBNA is proved to be an accurate, safe and minimally invasive procedure in the diagnosis of locoregional recurrence of surgically-treated lung cancer and should be considered as a first choice in patients with radiological abnormalities during follow up. EUS-B-FNA demonstrates to be useful in patients with locoregional lung cancer recurrence, not only in stations not reachable by EBUS-TBNA, but also in paratracheal nodes that could be better attained by the esophagus, whenever there are changes in the normal mediastinal architecture.

\section{Abbrevations \\ COPD: Chronic obstructive pulmonary disease; CT: Computed tomography; EBUS-TBNA: Endobronchial ultrasound transbronchial-guided needle aspiration; EUS-B-FNA: Endoscopic ultrasound fine-needle aspiration using an echobronchoscope; EUS-FNA: Endoscopic ultrasound fine-needle aspiration; FDG: Fludeoxyglucose; IQR: Interquartilic range; NPV: Negative predictive value; NSCLC: Non-small cell lung cancer; PET/CT: Positron emission tomography/ Computed tomography; PPV: Positive predictive value; SD: Standard deviation}

\section{Funding}

Dr. Felipe Andreo obtained a grant from the Spanish Society of Pulmonology (SEPAR).

\section{Availability of data and materials}

The database used for the study can be available from the corresponding author under demand if needed.

\section{Authors' contributions}

JSS Designed the study, performed EBUS-TBNA and EUS-B-FNA and wrote the manuscript. PS Designed the study, performed EBUS-TBNA and EUS-BFNA and wrote the manuscript. FA performed EBUS-TBNA and EUS-B-FNA and revised the manuscript. MT performed EBUS-TBNA and revised the manuscript. CC performed EBUS-TBNA and EUS-B-FNA and revised the manuscript. TM performed follow-up, selected patients and revised the manuscript. EC performed follow-up, selected patients and revised the manuscript. EF performed surgical procedures and revised the manuscript. SGR performed surgical procedures and revised the manuscript. JRM designed the study and revised the manuscript. All authors read and approved the final manuscript.

\section{Competing interests}

The authors declare that they have no competing interests.

\section{Consent for publication}

All the images used as figures in the manuscript were obtained once the patients gave the consent for publication.

\section{Ethics approval and consent to participate}

All patients signed the informed consend of the study that was approved by the local ethics committee (Comité de ética Hospital Germans Trias i Pujol).

\section{Author details}

'Pulmonology Department, Hospital Germans Trias i Pujol, Carretera de Canyet S/N. 08916, Badalona, Barcelona, Spain. ${ }^{2}$ Department de Medicina. Universitat Autònoma de Barcelona, Bellaterra, Barcelona, Spain. ${ }^{3}$ Catalan Institute of Oncology, Badalona, Barcelona, Spain. ${ }^{4}$ Thoracic Surgery Department, Hospital Germans Trias i Pujol, Badalona, Barcelona, Spain. 
Received: 14 December 2016 Accepted: 22 February 2017 Published online: 28 February 2017

\section{References}

1. Jemal A, Bray F, Center MM, et al. Global cancer statistics. CA Cancer J Clin. 2011:61:69-90

2. Sugimura H, Nichols FC, Yang P, Allen MS, Cassivi SD, Deschamps C, Williamb BA, Pairolero PC. Survival after recurrent nonsmall-cell lung cancer after compete pulmonary resection. Ann Thorac Surg. 2007;83:409-17.

3. Colt HG, Murgu SD, Korst RJ, Slatore CG, Unger M, Quadrelli S. Follow-up and surveillance of the patient with lung cancer after curative-intent therapy. Diagnosis and management of lung cancer, $3^{\text {rd }}$ ed: American College of Chest Physicians Evidence-Based Clinical Practice Guideliness. Chest. 2013;145(5 Suppl):e437S-54.

4. Zhou Q, West DG, Shelley-Frase G, Medford ARL. Foamy macrophage deposition in lymph nodes mimicking lung cancer recurrence diagnosed via endobronchial ultrasound-guided transbronchial needle aspiration. Respiration. 2015;90:426-9.

5. Hellwig D, Gröschel A, Graeter TP, Hellwig AP, Nestle U, Schäfers HJ, Sybrecht GW, Kirsch CM. Diagnostic performance and prognostic impact of FDG-PET in suspected recurrence of surgically treated non-small cell lung cancer. Eur J Nucl Med Mol Imaging. 2006:33:13-21.

6. Sudarski S, Henzler T, Schoenber SO. Post-therapeutic positron emission tomography/computed tomography for early detection of non-small cell lung cancer recurrence. Transl Lung Cancer Res. 2013;4:295-303.

7. Meersschaut D, Vermassen F, Brutel de la Riviere A, Knaepen PJ, Van den Bosch JM, Vanderschueren R. Repeat mediastinoscopy in the assessment of new and recurrent lung neoplasm. Ann Thorac Surg. 1992;53:120-2.

8. Leyn D, Dooms C, Kuzdzal J, Lardinois D, Passlick B, Rami-Porta R, Turna A, Van Schil P, Venuta F, Waller D, Weder W, Zielinski M. Revised ESTS guidelines for preoperative mediastinal lymph node staging for non-smallcell lung cancer. Eur J Cardiothorac Surg. 2014;45:787-98.

9. Vilmann P, Clementsen PF, Colella S, Siemsen M, De Leyn P, Dumonceau JM, Herth FJ, Larhi A, Vazquez-Sequeiros E, Hassan C, Crombag L, Korevaar DA, Konge L, Annema JT. Combined endobronchial and esophageal endosonography for the diagnosis and staging of lung cancer: European Society of Gastrointestinal Endoscopy (ESGE) Guideline, in cooperation with the European Respiratory Society (ERS) an the European Society of Thoracic Surgeons (ESTS). Endoscopy. 2015;47:545-59.

10. Hwangbo B, Lee HS, Lee GK, Lim KY, Lee SH, Lee HY, Lee JY, Zo J. Transoesophageal needle aspiration using a convex probe ultrasonic bronchoscope. Respirology. 2009;6:843-9.

11. Szlubowski A, Zielinski M, Soja J, Filarecka A, Orzechowski S, Pankowski J, Obrochta M, Wegrzyn J, Cmiel A. Accurate and safe mediastinal restaging by combined endobronchial and endoscopic ultrasound-guided needle aspiration performed by single ultrasound bronchoscope. Eur J Cardiothorac Surg. 2014;46:262-6.

12. Meena N, Hulet $C$, Jeffus $S$, Bartter $T$. Left adrenal biopsy using the convex curvilinear ultrasound scope. Respiration. 2015;89:57-61.

13. Crombag LMMJ, Annema JT. Left adrenal gland analysis in lung cancer patients using the endobronchial ultrasound scope: a feasibility trial. Crombag LMMJ, Annema JT. Respiration. 2016:91:235-40.

14. Anraku M, Pierre AF, Nakajima T, De Perrot M, Darling GE, Waddell TK, Keshavjee S, Yasufuku K. Endobronchial ultrasound-guided transbronchial needle aspiration in the management of previously treated lung cancer. Ann Thorac Surg. 2011;92:251-5.

15. Chen F, Miyahara R, Sato T, Sonobe M, Sakai H, Bando T, Date H. Usefulness of endobronchial ultrasound in patients with previously treated thoracic malignancy. Interact Cardiovasc Thorac Surg. 2012:14:34-7.

16. Han SG, Yoo H, Jhun BW, Park HY, Suh GY, Chung MP, Kim H, Kwon OJ, Han J, Um SW. The role of endobronchial ultrasound-guided transbronchial needle aspiration in the diagnosis of recurrent non-small cell lung cancer after surgery. Intern Med. 2013;52:1875-81.

17. Yamamoto T, Sakairi Y, Nakajima T, Suzuki H, Tagawa T, Iwata T, Mizobuchi T, Yoshida S, Nakatani Y, Yoshino I. Comparison between endobronchia ultrasound-guided transbronchial needle aspiration and ${ }^{18} \mathrm{~F}$-fluorodeoxyglucose positron emission tomography in the diagnosis of postoperative nodal recurrence in patients with lung cancer. Eur J Cardiothorac Surg. 2015:47:234-8.

18. Evison M, Crosbie PAJ, Califano R, Summers Y, Martin J, Barber PV, Booton R. Can EBUS-TBNA provide an accurate diagnosis in patients found to have enlarged or FDG-avid lymph nodes during surveillance of previously treated lung cancer? A retrospective study. J Bronchol Intervent Pulmonol. 2015;22: 114-20.

19. Du Rand IA, Blaikley J, Booton R, Chaudhuri N, Gupta V, Khalid S, Mandal S, Martin J, Mills J, Navani N, Rahman NM, Wrightson JM, Munayvar M, British Thoracic Society Bronchoscopy Guideline Group. British Thoracic Society guideline for diagnostic flexible bronchoscopy in adults: accredited by NICE. Thorax. 2013;68 Suppl 1:i1-44.

20. Alsharif M, Andrade RS, Groth S, Stelow EB, Pambuccian SE. Endobronchial ultrasound-guided transbronchial ultrasound-guided transbronchial fineneedle aspiration: the University of Minnesota experience, with emphasis on usefulness, adequacy assessment, and diagnostic difficulties. Am J Clin Pathol. 2008;130:434-43.

21. Martini N, Bains MS, Burt ME, Zakowski MF, McCormack P, Rusch WW, et al. Incidence of local recurrence and second primrary tumors in resected stage I lung cancer. J Thorac Cardiovasc Surg. 1995;109(1):120-9.

22. Fedor D, Johnson WR, Singhal S. Local recurrence following lung cancer surgery: incidence, risk factors, and outcomes. Surg Oncol. 2013;22:156-61.

23. Taylor MD, Nagji AS, Bhamidipati CM, Theodosakis N, Kozower BD, Lau CL, et al. Tumor recurrence after complete resection for non-samll cell lung cancer. Ann Thorac Surg. 2012;93(6):1813-21.

24. Louie BE, Kapur S, Farivar AS, Youssef SJ, Gorden J, Aye RW, Vallieres E. Safety and utility of mediastinoscopy in non-small cell lung cancer in a complex mediastinum. Ann Thorac Surg. 2011;92:278-83.

25. Call S, Rami-Porta R, Obiols C, Serra-Mitjans M, Gonzalez-Pont G, Bastus-Piulats R, Quintana S, Belda-Sanchis J. Repeat mediastinoscopy in all its indications: experience with 96 patients and 101 procedures. Eur J Cardiothorac Surg. 2011;39:1022-7.

26. Van Schil PE, De Waele M. A second mediastinoscopy: how to decide and how to do it? Eur J Cardiothorac Surg. 2008;33:703-6.

27. Yasufuku K, Nakajima T, Waddell T, et al. Endobronchial ultrasound-guided transbronchial needle aspiration for differentiating N0 versus N1 lung cancer. Ann Thorac Surg. 2013;96:1756-60.

28. Wada H, Hirohashi K, Nakajima T, Anayama T, Kato T, Grindlay A, McConnell J, Yoshino I, Yasufuku K. Assessment of the new thin convex probe endobronchial ultrasound bronchoscope and the dedicated aspiration needle: a preliminary study in the porcine lung. J Bronchology Interv Pulmonol. 2015:22:20-7.

29. Zielinski M, Szlubowski A, Kolodziej M, Orzechowski S, Laczynska E, Pankowski J, Jakubiak M, Obrochta A. Comparison of endobronchial ultrasound and/or endoesophageal ultrasound with transcervical extended mediastinal lymphadenectomy for staging and restaging of non-small-cell lung cancer. J Thorac Oncol. 2013;8:630-6.

30. Herth FJ, Annema JT, Eberhardt R, Yasufuku K, Ernst A, Krasnik M, Rintoul RC. Endobronchial ultrasound with transbronchial needle aspiration for restaging the mediastinum in lung cancer. J Clin Oncol. 2008;26:3346-50.

\section{Submit your next manuscript to BioMed Central and we will help you at every step:}

- We accept pre-submission inquiries

- Our selector tool helps you to find the most relevant journal

- We provide round the clock customer support

- Convenient online submission

- Thorough peer review

- Inclusion in PubMed and all major indexing services

- Maximum visibility for your research

Submit your manuscript at www.biomedcentral.com/submit
) Biomed Central 